\title{
Congenital Hemangiopericytoma
}

\author{
FAUZIAJ AHAN ${ }^{1}$, PK GHOSH $^{2}$, REHANA KHANAM ${ }^{1}$, SAIDUR RAHMAN ${ }^{3}$
}

\begin{abstract}
Hemangiopericytoma is a rare vascular neoplasm that usually occurs in adults. Occurrence of the tumors in infants, known as congenital or infantile hemangiopericytoma, is rare and its behavior is different than that of adult type. We are reporting a 10 day old male neonate presenting with a mass on his left arm since birth. Clinically it was suspected as lymphangioma because of its high incidence in pediatric patients. The tumor was surgically removed and histopathologically diagnosed as a case of hemangiopericytoma.
\end{abstract}

Keyword: Hemangiopericytoma, congenital type.

\section{Introduction}

Haemangiopericytoma is a rare vascular tumor first described by Stout and Murray in $1942 .{ }^{1}$ Only $10 \%$ cases occur in children less than 15 years of age. ${ }^{2}$ Kauffman and Stout stated that the character of tumor is more benign in children less than 1 year than in adult. ${ }^{3}$ The incidence is higher in boys than in girls. ${ }^{3}$ Histopathologically, it often shows increased cellularity, prominent mitotic activity, foci of necrosis and hemorrhage. These histopathological findings are similar to malignant hemangiopericytoma in adults. ${ }^{4}$

Thus, congenital hemangiopericytoma is an interesting tumor because of its dissociation between the malignant histopathological findings and benign clinical course.

\section{Case report:}

A 10 day old male baby was admitted in neonatal ward with a mass on his right side of upper arm. This mass was present since birth. On admission, physical examination revealed presence of a mass measuring $6 \mathrm{X} 4 \mathrm{~cm}$. It was firm and nonmobile. No obvious color change was noticed over the skin. Before surgery certain routine investigations were performed and all of these were within normal range. The mass was removed surgically. Immediate postoperative outcome was uneventful but the patient was not available to assess remote postoperative outcome as the parents abruptly left the hospital.

After surgery naked eye examination of the specimen revealed a $5 \mathrm{X} 4 \mathrm{X} 3.5 \mathrm{~cm}$, tan gray skin covered mass. Histopathological examination showed a highly cellular tumour composed of slender, fusiform cells with indistinct cytoplasmic border. These were arranged in sheets and tightly packed clusters mostly disposed around ramifying

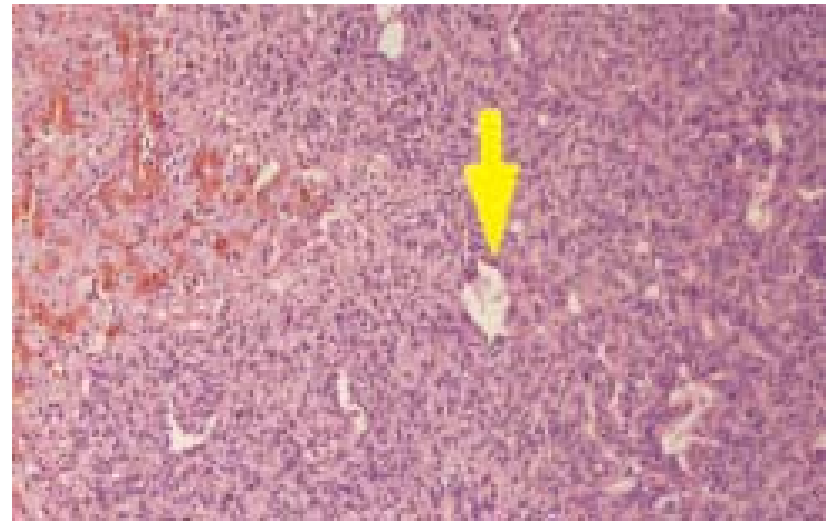

Fig.-1: Hematoxylin and eosin stain of show highly cellular tumour with staghorn type of blood vessels.

blood vessels, lined by single layer of endothelial cells. In some areas these spaces showed stag-horn appearance. Focal areas of haemorrhage and degenerative changes were also present. Mitotic figures were scanty.

\section{Discussion:}

Hemangioperictyoma (HPC) has a wide variety of presentation in terms of tumour size, pattern of growth, clinical presentation and malignant potential. ${ }^{5}$ Numerous studies have been described regarding the clinical and pathological features of HPC since its first description in 1942. ${ }^{1}$ Two distinct entities have been described. The adult type occurs in adults and in children older than one year and the congenital type which occurs in the first year of life. ${ }^{6}$

Most of the congenital tumours are benign in nature. The reasons for the benign characteristics of congenital HPC are not well understood. ${ }^{7}$ However, children over one year have similar malignant potential like adults. Increased age is a risk

1. Assistant Professor, Department of Pathology, Bangladesh Medical College.

2. Professor and Head, Department of Pathology, Bangladesh Medical College.

3. Associate Professor, Department of Pathology, Bangladesh Medical College.

Correspondence: Dr. Fauzia Jahan, Assistant Professor, Department of Pathology, Bangladesh, Medical College. 
factor associated with a more malignant course ${ }^{2,5}$ Congenital HPC is more specialized than those of adult type because of clinical and histological features. ${ }^{9}$ Its clinical behavior is also better than that of adult type with reports of spontaneous regression of the tumor. ${ }^{10}$

It is predominantly located subcutaneously mostly in head and neck region. This displays an indolent behavior despite worrisome histologic features such as extensive hemorrhage, necrosis, hypercellularity and increased mitotic figures. ${ }^{8}$ Histologically congenital HPC is multilobulated and has a more prominent collagenous matrix. The distribution of vessel is more irregular than adult form. The immunohistochemical staining of muscle related actin, a feature of normal pericyte is generally observed in congenital HPC, whereas this protein is rarely seen in that of adults. ${ }^{9}$

The diagnosis is mostly dependant on the histopathology. Imaging studies like MRI, CT scan and laboratory examination are seldom helpful. ${ }^{7}$ Other techniques like angiogram are nonspecific but the informations obtained from these may be useful in preoperative planning of surgery. ${ }^{7}$

Commonly the management of congenital HPC is by local excision. In case of incomplete excision, chemotherapy or radiotherapy is recommended. ${ }^{6}$ However, in rare instances, true malignant congenital HPC have been reported. ${ }^{11}$

Good responses to therapy are common. No recurrences with residual tumor have been reported in the infantile type. 11-15 Considering altogether it should be mentioned here that inappropriately aggressive therapy should be avoided and long-term follow-up is necessary.

\section{Conclusion:}

Congenital hemangiopericytoma is a rare vascular neoplasm that should be considered in the differential diagnosis of vascular malformation in pediatric patient. Diagnosis requires biopsy and histological confirmation. The histological behavior is different from the adult type regarding the aggressiveness of the tumor, recurrence and malignant potential. Chemotherapy may be considered for unresectable lesion, recurrence or in case of metastasis. Long-term follow up in these cases are mandatory.

\section{Conflict of Interest: None}

\section{References:}

1. Stout AP, Murray MR. Haemangiopaericytoma: a vascular tumour featuring Zimmermann's pericytes. Ann Surg 1942; 116:26-33
2. Chung EB. Pitfalls in diagnosing benign soft tissue tumors in infancy and childhood. Ann Pathol 1985; 20:323-86

3. Kauffman SL, Stout AP.Hemangiopericytoma in children. Cancer1960; 13:695-710

4. Seisuke S, Morihiro S, Miwako N, Tatsuo K et al. A Case of Congenital Hemangiopericytoma Journal of the Japanese Society of Pediatric Surgeons 1999 ;35(.2): 231-236

5. Del Rosario ML, Saleh A. preoperative chemotherapy for congenital hemangiopericytoma and a review of the literature.J Pediatr Hematol Oncol 1997; 19:247-50

6. Coffin CM. Vascular tumors. In: Coffin CM, Dehner LP, O’Shea PA, editors. Pediatric Soft Tissue Tumors. A clinical, pathological, and therapeutic approach. 1st ed. Baltimore: Williams and Wilkins, 1997:40-79.

7. Hoey SA, Letts RM, Jimenez C. Infantile hemangiopericytoma of musculoskeletal system: case reports and literature review. J Pediatr Orthorp 1998; 18:359-62

7. Enzinger FM, Smith BH. Hemangiopericytoma: and analysis of 106 cases. Hum pathology 1976; 7:61-82

8. Kowalski PJ, Paulino AFG. Proliferation index as a prognostic marker in hemangiopericytoma of the head and neck. Head \& Neck 2001; 23: 492-496

9. Morgan A, Evbuomwan I. Congenital hemangiopericytoma of the face with early distant metastasis. J R Coll Surg Edinb 1983; 28:123-5.

10. Nappi O, Ritter JH, Pettinato G et al. Hemangiopericytoma: histopathological pattern or clinicopathologic entity? Semin Diagn Pathol 1995; 12:221-232

11. Jenkins JJ III. Congenital malignant hemangiopericytoma. Pediatr Path 1987; 7:119-122

12. Chung KC, Weiss SW, Kuzon WM, Multifocal congenital hemangiopaericytoma associated with Kasabach-Merritt syndrome. Br J Plast Surg1995; 48:240-2

13. Toren A, Perimen Mpolak-Charcon S et al. Congenital hemangiopericytoma/infantile myofibromatosis: radical surgery versus a conservative” wait and see”approach. Pediatr Hematol Oncol 1997; 14:3877-93

14. Rodrguez-Galindo C, Ramsey K, Jenkins JJ,et al. Hemangiopericytoma in children and infants. Cancer 2000; 88:198-204 